\title{
The Impact of Extent of Liver Resection Among Patients with Neuroendocrine Liver Metastasis: an International Multi-institutional Study
}

\author{
Jonathan G. Sham ${ }^{1} \cdot$ Aslam Ejaz ${ }^{1} \cdot$ Michele M. Gage $^{1} \cdot$ Fabio Bagante $^{2} \cdot$ Bradley N. Reames $^{1} \cdot$ Shishir Maithel $^{3}$. \\ George A. Poultsides ${ }^{4} \cdot$ Todd W. Bauer $^{5} \cdot$ Ryan C. Fields $^{6} \cdot$ Matthew J. Weiss $^{1} \cdot$ Hugo Pinto Marques ${ }^{7}$. \\ Luca Aldrighetti $^{8} \cdot$ Timothy M. Pawlik $^{2} \cdot \mathrm{Jin} \mathrm{He}^{1}$
}

Received: 5 March 2018 / Accepted: 22 June 2018 / Published online: 6 July 2018

(C) 2018 The Society for Surgery of the Alimentary Tract

\begin{abstract}
Background Liver resection in patients with neuroendocrine liver metastasis (NELM) provides a survival benefit, yet the optimal extent of resection remains unknown. We sought to examine outcomes of patients undergoing non-anatomic (NAR) versus anatomic liver resection (AR) for NELM using a large international cohort of patients.

Methods Two hundred and fifty-eight patients who underwent curative intent liver resection from January 1990 to December 2016 were identified from eight institutions. Patients were excluded if they underwent concurrent ablation, had extrahepatic disease, underwent a debulking operation, or had mixed anatomic and non-anatomic resections. Overall (OS) and recurrence-free (RFS) survival were compared among patients based on the extent of liver resection (AR vs. NAR).

Results Most primary tumors were located in the pancreas $(n=117,45.4 \%)$ or the small intestine $(n=65,25.2 \%)$. Liver resection consisted of NAR $(n=126,48.8 \%)$ or AR $(n=132,51.2 \%)$ resection. The overwhelming majority of patients who underwent NAR had an estimated liver involvement of $<50 \%$ (NAR $109,97.3 \%$ vs. AR $n=82,65.6 \% ; P<0.001$ ). Patients who underwent NAR also had higher rates of primary tumor lymph node metastasis (NAR $n=79,71.2 \%$ vs. AR $n=37,33.6 \% ; P<0.001$ ) and microscopically positive margins (R1) (NAR $n=29,25.7 \%$ vs. AR $n=16,12.5 \% ; P=0.009$ ). After a median follow-up of 47.7 months, $48(18.6 \%)$ patients died and 37.0\% $(n=95)$ had evidence of disease recurrence. Patients who underwent AR had both longer median OS (not reached) and RFS (not reached) versus patients who underwent NAR (median OS 138.3 months; median RFS 31.3 months) (both $P<0.01$ ). After controlling for patient and disease-related factors, extent of liver resection was independently associated with an increased risk of recurrence (HR 2.39, 95\% CI 1.04-5.48; $P=0.04$ ) but not death (HR 1.92, 95\% CI 0.40-9.28; $P=0.42$ ).

Conclusion NAR was independently associated with a higher incidence of recurrence versus patients who undergo a formal anatomic hepatectomy among patients with NELM.
\end{abstract}

Keywords Neuroendocrine tumors $\cdot$ Hepatectomy $\cdot$ Anatomic resection

Jin He

jhe11@jhmi.edu

1 Department of Surgery, The Johns Hopkins University School of Medicine, 600 N. Wolfe Street, Halsted 614, Baltimore, MD 21287, USA

2 Division of Surgical Oncology, The Ohio State University Comprehensive Cancer Center, Columbus, OH, USA

3 Department of Surgery, Emory University, Atlanta, GA, USA
4 Department of Surgery, School of Medicine, Stanford University, Stanford, CA, USA

5 Department of Surgery, University of Virginia, Charlottesville, VA, USA

6 Department of Surgery, School of Medicine, Washington University, St. Louis, MO, USA

7 Department of Surgery, Curry Cabral Hospital, Lisbon, Portugal

8 Department of Surgery, Liver Unit, Scientific Institute San Raffaele, Vita-Salute San Raffaele University, Milan, Italy 


\section{Background}

Neuroendocrine tumors are a group of generally indolent epithelial neoplasms that have the ability to metastasize. The liver is the most common site of spread, with liver metastasis ultimately developing in $60-90 \%$ of patients. ${ }^{1}$ Neuroendocrine liver metastases (NELM) are the primary source of morbidity in patients with these cancers and can lead to local and systemic symptoms, liver failure, and death. The presence of NELM portends worse survival compared with patients without NELM ${ }^{2}$ however, improvement has been demonstrated with appropriately selected liver-directed therapies. ${ }^{3}$ Only $20 \%$ of patients with NELM will be candidates for curative intent resection at the time of diagnosis due to the size and locations of metastases, as well as the frequency of multiple tumors and bilobar disease. ${ }^{4}$

Among patients undergoing resection for NELM, data are lacking regarding oncologic outcomes associated with anatomic resection (AR) versus non-anatomic surgical or "wedge" resection (NAR). In the setting of colorectal liver metastases (CRLM), parenchyma-preserving NAR has been shown to decrease morbidity without sacrificing oncologic effectiveness. ${ }^{5}$ However, for certain subtypes of CRLM, NAR has been associated with worse disease free survival ${ }^{6}$ and inferior oncologic outcomes. ${ }^{7}$ For NELM, multiple studies have demonstrated improved survival when comparing surgery with medical or catheter-based therapies ${ }^{8,9}$ and reasonable survival even when only debulking hepatic disease. ${ }^{10}$ Despite the established role for surgery in the setting of NELM, the relative efficacy of AR and NAR for NELM is not known. As such, we sought to examine the long-term outcomes of patients with NELM who underwent hepatectomy stratified by type of hepatectomy (NAR vs. AR) using a large multi-institutional international.

\section{Methods}

\section{Patient Selection and Data Collection}

All patients who underwent liver resection for NELM between January 1990 and December 2016 were identified from an international multi-institutional database. This multiinstitutional database included patients treated at eight major hepatobiliary institutions (The Ohio State University Comprehensive Cancer Center, Columbus, OH; Johns Hopkins Hospital, Baltimore, MD; Stanford University, Stanford, CA; Washington University School of Medicine, St Louis, MO; University of Virginia, Charlottesville, VA; Scientific Institute San Raffaele, Vita-Salute San Raffaele University, Milan, Italy; Curry Cabral Hospital, Lisbon, Portugal; Winship Cancer Institute, Emory University, Atlanta, GA) as previously described. ${ }^{11}$ Patients were excluded if they underwent concurrent ablation, had extrahepatic disease, underwent a debulking operation, or had mixed anatomic and non-anatomic resections. Patients were stratified by extent of liver resection (AR vs. NAR). A non-anatomical liver resection is defined as any parenchymal-sparing liver resection that did not include $>2$ contiguous liver resection. The Institutional Review Board of the participating institutions approved the study.

Standard demographic and clinicopathologic data were collected including age, gender, race, type of surgery, and tumor- specific characteristics of both the primary NET and the liver metastases. Tumor-specific characteristics of the primary neuroendocrine tumor included location, histology, tumor functional status, grade of differentiation, and presence or absence of lymph node metastases. Ki-67 index levels and mitoses/hpf were not universally collected and therefore not included in analysis. Grade of tumor differentiation was defined according to the 2010 WHO grading system: G1, well differentiated; G2, moderately differentiated; and G3, poorly differentiated. ${ }^{12}$ The updated 2017 WHO grading classification was not used due to incomplete Ki67 and mitoses/hpf data available for these patients. Data on treatment-related variables, such as type of liver surgery, resection margin, and rate of liver involvement, were collected. An R0 resection was defined as a microscopically negative margin on final pathology $(>1 \mathrm{~mm})$, and an $\mathrm{R} 1$ resection was defined as a microscopically positive margin on final pathology without any known gross residual disease. The primary outcome of interest was overall survival (OS) defined as the time interval between the date of surgery and the date of death. Recurrence-free survival (RFS) was similarly calculated as the interval between date of surgery and the date of first evidence of tumor recurrence. Surveillance protocols were left to the discretion of the surgeon and medical oncologist.

\section{Statistical Analysis}

Discrete variables were reported as medians with interquartile range (IQR); categorical variables were reported as totals and frequencies. Univariable comparisons were assessed using the chi-squared or Wilcoxon-rank sum test as appropriate. Overall survival time was calculated from the date of initial liver resection. Survival adjusted for censoring was calculated using the Kaplan-Meier method and median values were compared using the log-rank test. The impact of various clinicopathological factors on OS and RFS was assessed using a Cox proportional hazards model. All analyses were carried out with STATA version 13.0 (StataCorp, College Station, TX), and a $P$ value of $<0.05$ (two-tailed) was considered statistically significant. 


\section{Results}

Two hundred and fifty-eight patients who underwent surgical resection for NELM and met inclusion criteria were included in the analytic cohort (Table 1). Most patients were treated after the year $2000(n=224,86.8 \%)$. Median patient age was 57 (IQR 48, 65). Most patients were Caucasian ( $n=$ $217,85.4 \%)$ and female $(n=141,54.6 \%)$. Among the patients with a known primary tumor location, most tumors originated in the pancreas $(n=117,45.4 \%)$, with the small intestine $(n=$ $65,25.2 \%$ ) being the next most common site. Patients underwent a variety of operations for extirpation of the primary tumor including a pancreatic resection $(46.6 \%)$ or intestinal resection $(29.7 \%)$. Prior to liver resection, 35 patients $(13.6 \%)$ received systemic octreotide therapy whereas 18 patients (7.0\%) received chemotherapy. Unfortunately the type and duration of chemotherapy was not recorded. Patients underwent liver resection at a median of 24 months (IQR $9.9,55.5)$ from the date of primary tumor resection.

The type of liver resection was nearly evenly split between NAR $(n=126,48.8 \%)$ and AR $(n=132,51.2 \%)$. The rates of AR differed among the eight institutions (26.8-100\%). One institution performed all 28 resections with an AR. There was no difference in patient age comparing patients who underwent
NAR (57, IQR 49, 65) versus AR (56.5, IQR 45, 66; $P=0.78)$. Similarly, the incidence of symptomatic disease (NAR $n=76$, $60.3 \%$ vs. AR $n=78,59.2 \% ; P=0.84)$ and bilateral liver metastases (NAR $n=64,30.2 \%$ vs. AR $n=38,31.4 \% ; P=0.66$ ) was equivalent between the two groups.

Among the 224 patients for which final pathologic tumor grade was known, no differences were observed between the NAR and AR groups. Patients undergoing NAR had higher rates of lymph node metastases (NAR $n=79,71.2 \%$ vs. AR $n=37,33.6 \% ; P<0.001)$ as well as estimated liver involvement of $<50 \%$ (NAR $n=191,97.3 \%$ vs. AR $n=82,65.6 \%$; $P<0.001)$. Patients who underwent NAR had a median number of two lesions resected (IQR 1, 3). With regard to resection margin status, NAR was associated with lower rates of an R0 resection (NAR $n=84,74.3 \%$ vs. AR $n=112,87.5 \% ; P=$ $0.009)$. Following liver resection, 51 patients $(19.8 \%)$ received octreotide therapy and 21 patients $(8.1 \%)$ received chemotherapy.

\section{Factors Affecting Overall Survival}

After a median follow-up of 47.7 months, 48 (18.6\%) patients in the entire cohort had died and $95(37.0 \%)$ had evidence of disease recurrence. The 1-, 3-, and 5-year overall survival
Table 1 Clinicopathological characteristics of patients who underwent resection for neuroendocrine liver metastasis

\begin{tabular}{|c|c|c|c|c|}
\hline & $\begin{array}{l}\text { All patients } \\
(N=258)\end{array}$ & $\begin{array}{l}\text { Non-anatomic resection } \\
(N=126)\end{array}$ & $\begin{array}{l}\text { Anatomic resection } \\
(N=132)\end{array}$ & $P$ value \\
\hline Age, years (IQR) & $57(48,65)$ & $57(49,65)$ & $56.5(45,66)$ & 0.78 \\
\hline Male sex & $117(45.4)$ & $57(45.2)$ & $60(45.5)$ & 0.97 \\
\hline Ethnicity & & & & 0.43 \\
\hline Caucasian & $217(85.4)$ & $103(83.7)$ & $114(87.0)$ & \\
\hline Black & $25(9.8)$ & $12(9.8)$ & $13(9.9)$ & \\
\hline Other & $12(4.7)$ & $8(6.5)$ & $4(3.1)$ & \\
\hline Location of primary tumor & & & & 0.003 \\
\hline Pancreas & $117(45.4)$ & $66(52.4)$ & $51(38.6)$ & \\
\hline Small Intestine & $65(25.2)$ & $38(30.2)$ & $27(20.5)$ & \\
\hline Symptomatic disease & $154(59.7)$ & $76(60.3)$ & $78(59.1)$ & 0.84 \\
\hline Primary tumor grade $(N=224)$ & & & & 0.99 \\
\hline Low & $106(61.3)$ & $51(60.7)$ & $55(61.8)$ & \\
\hline Intermediate & $51(29.5)$ & $25(29.8)$ & $26(29.2)$ & \\
\hline High & $16(9.3)$ & $8(9.5)$ & $8(9.0)$ & \\
\hline Lymph node metastasis $(N=221)$ & $116(52.5)$ & $79(71.2)$ & $37(33.6)$ & $<0.001$ \\
\hline Synchronous liver metastasis & $151(58.5)$ & $81(64.3)$ & $70(53.0)$ & 0.07 \\
\hline Bilateral liver metastases & $64(30.2)$ & $38(31.4)$ & $26(28.6)$ & 0.66 \\
\hline Estimated liver involvement & & & & $<0.001$ \\
\hline$<50 \%$ & $191(80.6)$ & $109(97.3)$ & $82(65.6)$ & \\
\hline$\geq 50 \%$ & $46(19.4)$ & $3(2.7)$ & $43(34.4)$ & \\
\hline Resection margin status & & & & 0.009 \\
\hline R0 & $196(81.3)$ & $84(74.3)$ & $112(87.5)$ & \\
\hline $\mathrm{R} 1$ & $45(18.7)$ & $29(25.7)$ & $16(12.5)$ & \\
\hline
\end{tabular}


(OS) was $95.9,92.3$, and $84.4 \%$, respectively. No patient factors (age, race, gender) were associated with worse median OS (Table 2). Compared with patients who had pancreatic tumors, patients with primary tumors of the small intestine demonstrated longer OS (pancreas, 151 months vs. small intestine, not reached, $P=0.006$ ). Pathologic factors associated with worse OS included intermediate-high-grade tumors (low grade, not reached vs. intermediate-high grade, 151 months; $P=0.04$ ) and lymph node metastasis (no lymph node metastasis, not reached vs. lymph node metastasis, 138.3 months; $P=0.001)$. The presence of synchronous, bilateral, and extensive liver involvement $\geq 50 \%$ did not affect OS on multivariable analysis (all $P>0.05$ ). Furthermore, no difference was observed in OS comparing NAR versus AR (HR 0.94, 95\% CI 0.12-7.44, P=0.96) (Fig. 1). However, a resection margin status of R0 was independently associated with prolonged OS on Cox proportional hazard regression analysis (HR 2.92, 95\% CI 1.65-5.17, P<0.001).
Table 2 Hazard regression analysis of factors associated with overall survival

\begin{tabular}{|c|c|c|c|c|c|}
\hline \multirow[b]{2}{*}{ Variables } & \multirow[b]{2}{*}{$\begin{array}{l}\text { Median survival } \\
\text { (months) }\end{array}$} & \multirow[b]{2}{*}{$P$ value } & \multicolumn{3}{|c|}{ Multivariable survival analysis } \\
\hline & & & Hazard ratio & $95 \% \mathrm{CI}$ & $P$ value \\
\hline Age & & 0.50 & & & \\
\hline$<65$ years & Not reached & & Ref & & \\
\hline$\geq 65$ years & Not reached & & 1.28 & $0.75-2.18$ & 0.36 \\
\hline Race & & 0.28 & & & \\
\hline White & Not reached & & & & \\
\hline Black & 88.1 & & & & \\
\hline Other & 86.2 & & & & \\
\hline Gender & & 0.93 & & & \\
\hline Male & Not reached & & & & \\
\hline Female & Not reached & & & & \\
\hline Location of primary tumor & & 0.06 & & & \\
\hline Pancreas & 151.0 & & Ref & - & \\
\hline Small intestine & Not reached & & 0.39 & $0.19-0.76$ & 0.006 \\
\hline Large intestine & Not reached & & 0.70 & $0.30-1.61$ & 0.40 \\
\hline Symptomatic disease & Not reached & 0.24 & 0.76 & $0.42-1.39$ & 0.38 \\
\hline Primary tumor grade & & 0.11 & & & \\
\hline Low & Not reached & & Ref & - & \\
\hline Intermediate & 151.02 & & 1.92 & $1.04-3.56$ & 0.04 \\
\hline High & Not reached & & 1.55 & $0.73-3.29$ & 0.25 \\
\hline Lymph node status & & 0.001 & & & \\
\hline No lymph node metastasis & Not reached & & Ref & - & \\
\hline Lymph node metastasis & 138.3 & & 1.95 & $1.01-3.76$ & 0.05 \\
\hline Liver disease presentation & & 0.11 & & & \\
\hline No synchronous disease & Not reached & & Ref & - & \\
\hline Synchronous disease & Not reached & & 1.27 & $0.68-2.83$ & 0.35 \\
\hline Liver metastasis location & & 0.79 & & & \\
\hline Unilateral metastases & 138.3 & & & & \\
\hline Bilateral metastases & 124.4 & & & & \\
\hline Estimated liver involvement & & 0.003 & & & \\
\hline$<50 \%$ & 151.0 & & Ref & - & \\
\hline$\geq 50 \%$ & Not reached & & 1.40 & $0.69-2.83$ & 0.94 \\
\hline Type of liver resection & & 0.005 & & & \\
\hline Non-anatomic & 138.3 & & Ref & - & \\
\hline Anatomic & Not reached & & 0.94 & $0.12-7.44$ & 0.96 \\
\hline Resection margin & & 0.04 & & & \\
\hline R0 & Not reached & & & & \\
\hline $\mathrm{R} 1$ & 151.02 & & 2.92 & $1.65-5.17$ & $<0.001$ \\
\hline
\end{tabular}


Fig. 1 Kaplan-Meier survival curves comparing overall survival in patients undergoing anatomic and non-anatomic hepatic resections for NELM

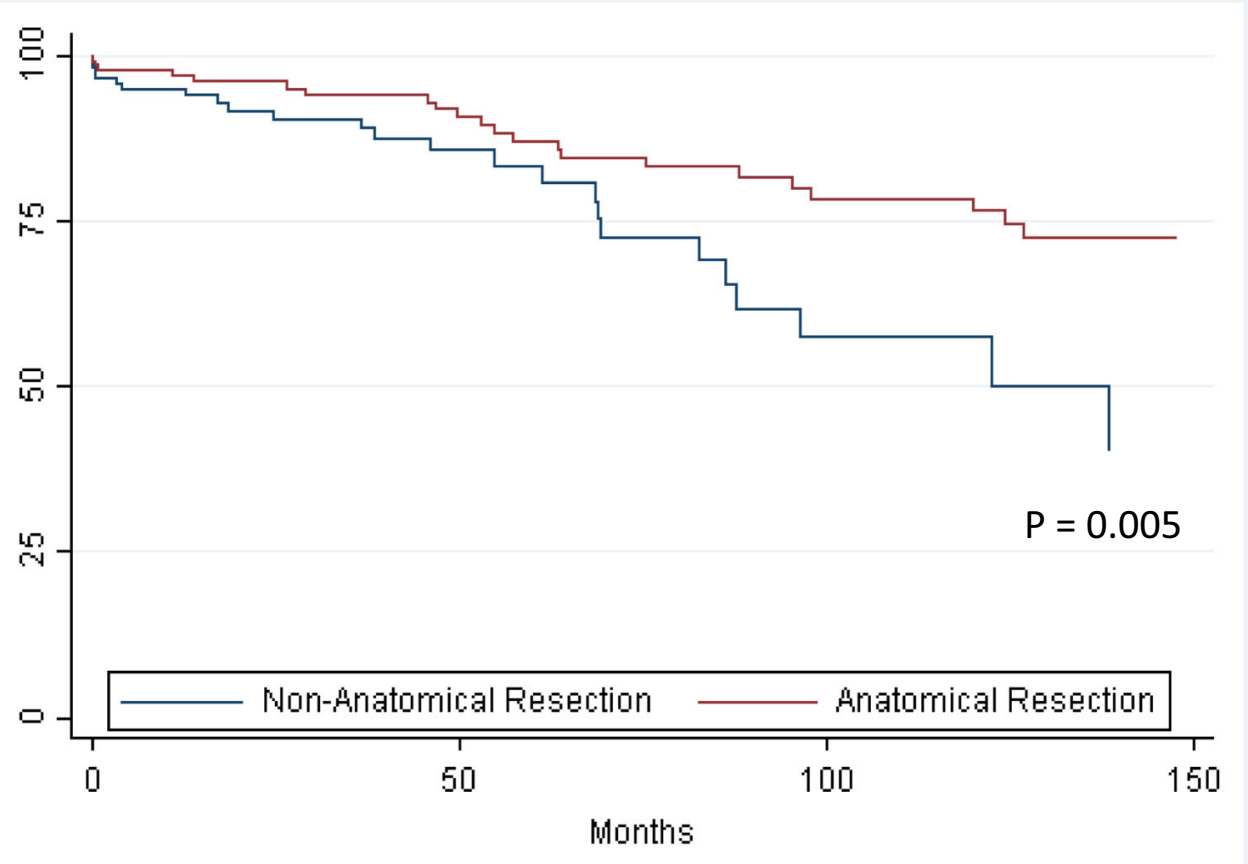

\section{Factors Affecting Recurrence-Free Survival}

Disease recurrence occurred in 95 (37.0\%) patients, with all but 6 patients experiencing a recurrence in the liver $(n=89,93.7 \%)$. Several clinicopathological factors were associated with differences in RFS in the cohort. Patients younger than 65 years of age had shorter median RFS as compared to patients greater than 65 years of age $(<65$ years, 40 months vs. $\geq 65$ years, not reached; $P=0.001$ ) (Table 3). Similar to differences noted in OS, patients who had primary pancreatic neuroendocrine tumors also had shorter median RFS (pancreas 31.3 months vs. small intestine 65.1 months; $P=0.004$ ) versus patients with mid- or hind-gut tumors. Pathologic factors associated with worse RFS included intermediate-high-grade tumors (low grade, not reached vs. intermediate-high grade, 56 months; $P=0.02$ ) and metastatic lymph node status (no lymph node metastasis, not reached vs. lymph node metastasis, 31.4 months; $P<0.001)$. Similarly, patients with synchronous liver disease at presentation (no synchronous disease 122.4 months vs. synchronous disease 54.1 months; $P=0.003$ ), an estimated liver involvement of $<50 \%(<50 \%, 44.3$ months vs. $\geq 50 \%$, not reached; $P<0.001$ ), R1 resection margin status (R0 95.2 months vs. R1 26.1 months; $P<0.001$ ), and patients who underwent a NAR (NAR, 31.3 months vs. AR, not reached; $P<0.001$ ) had a shorter RFS. After controlling for other measurable factors, multivariable analysis of low primary tumor grade (HR 2.56, 95\% CI 1.26-5.61; $P=0.01$ ) and R0 margin status (HR 3.22, $95 \%$ CI $1.33-7.82, P=0.01$ ) remained independently associated with longer RFS. Additionally, patients who underwent AR lived longer without recurrence than patients who underwent NAR (HR 2.39, 95\% CI 1.04-5.48, $P=0.04$ ) (Fig. 2).

\section{Discussion}

Surgery plays a vital role in the treatment of both localized and metastatic neuroendocrine malignancies, regardless of the site of primary tumor. ${ }^{8,9}$ With regard to patients with liver metastasis, several previous studies have demonstrated improved survival with aggressive treatment of NELM. ${ }^{13}$ Even in cases where complete hepatic cytoreduction cannot be achieved, cytoreductive liver debulking operations have been associated with a survival benefit. ${ }^{10}$ Among patients with resectable hepatic disease, however, no study to our knowledge has evaluated the impact of the extent of liver resection on long-term outcomes. In contrast, several studies suggest that NAR provides similar oncologic outcomes compared with formal $A R$ with the advantage of decreasing perioperative morbidity among patients with colorectal liver metastasis. ${ }^{5}$ In the current study, we present one of the largest series comparing the oncologic outcomes of patients with NELM following AR and NAR. In concordance with previous studies, we found that an $\mathrm{R} 1$ resection margin status significantly decreased OS and RFS, while the amount of estimated liver involvement and the presence of bilateral liver disease did not. Perhaps more importantly, we noted that patients who underwent NAR for their NELM experienced a $>2$-fold risk of recurrence compared to patients receiving AR after adjusting for pertinent covariates.

Among patients with NELM, the best hope for long-term survival is complete surgical excision of all known primary and metastatic disease. For various reasons, including tumor location, tumor size, background hepatic function, estimated future liver remnant, and patient functional status, surgeons 
Table 3 Hazard regression analysis of factors associated with recurrence-free survival

\begin{tabular}{|c|c|c|c|c|c|}
\hline \multirow[b]{2}{*}{ Variables } & \multirow[b]{2}{*}{$\begin{array}{l}\text { Median survival } \\
\text { (months) }\end{array}$} & \multirow[b]{2}{*}{$P$ value } & \multicolumn{3}{|c|}{ Multivariable survival analysis } \\
\hline & & & Hazard ratio & $95 \% \mathrm{CI}$ & $P$ value \\
\hline Age & & 0.001 & & & \\
\hline$<65$ years & 40.0 & & Ref & & \\
\hline$\geq 65$ years & Not reached & & 0.28 & $0.12-0.66$ & 0.003 \\
\hline Race & & 0.20 & & & \\
\hline White & 63.6 & & & & \\
\hline Black & 33.0 & & & & \\
\hline Other & 11.5 & & & & \\
\hline Gender & & 0.87 & & & \\
\hline Male & 58.4 & & & & \\
\hline Female & 62.9 & & & & \\
\hline Location of primary tumor & & 0.004 & & & \\
\hline Pancreas & 31.3 & & Ref & - & \\
\hline Small intestine & 65.1 & & 0.15 & $0.06-0.39$ & $<0.001$ \\
\hline Large intestine & Not reached & & - & - & - \\
\hline Symptomatic disease & 44.31 & 0.03 & 1.25 & $0.57-2.75$ & 0.58 \\
\hline Primary tumor grade & & 0.05 & & & \\
\hline Low & Not reached & & Ref & - & \\
\hline Intermediate & 54.8 & & 2.09 & $0.92-4.77$ & 0.08 \\
\hline High & Not reached & & 5.99 & $1.68-21.38$ & 0.006 \\
\hline Lymph node status & & $<0.001$ & & & \\
\hline No lymph node metastasis & Not reached & & Ref & - & \\
\hline Lymph node metastasis & 31.4 & & 1.15 & $0.49-2.73$ & 0.75 \\
\hline Liver disease presentation & & 0.003 & & & \\
\hline No synchronous disease & 122.4 & & Ref & - & \\
\hline Synchronous disease & 54.1 & & 1.42 & $0.61-.3 .29$ & 0.41 \\
\hline Liver metastasis location & & 0.76 & & & \\
\hline Unilateral metastases & 37.6 & & Ref & - & - \\
\hline Bilateral metastases & 38.2 & & 1.02 & $0.42-2.48$ & 0.97 \\
\hline Estimated liver involvement & & $<0.001$ & & & \\
\hline$<50 \%$ & 44.3 & & Ref & - & \\
\hline$\geq 50 \%$ & Not reached & & 1.12 & $0.28-4.47$ & 0.87 \\
\hline Type of liver resection & & $<0.001$ & & & \\
\hline Non-anatomic & 31.3 & & Ref & - & \\
\hline Anatomic & Not reached & & 2.39 & $1.04-5.48$ & 0.04 \\
\hline Resection margin & & $<0.001$ & & & \\
\hline R0 & 95.2 & & & & \\
\hline $\mathrm{R} 1$ & 26.1 & & 3.22 & $1.33-7.82$ & 0.01 \\
\hline
\end{tabular}

may choose either AR or NAR. In the current cohort of over 250 patients, patients were evenly split between the types of surgical resection performed. Of note, patients who underwent AR had a higher rate of pancreas as the primary tumor location, which was independently associated with worse RFS and OS. Patients who underwent NAR were also more than twice as likely to harbor lymph node metastases after resection of the primary tumor. This potentially represents more aggressive disease biology that may have impacted the surgeon's decision to offer NAR as opposed to AR. However, this difference was observed despite almost an equal distribution between low, intermediate, and high-grade tumors between the two groups. With regard to immediate surgical oncologic outcomes, patients who underwent NAR were twice as likely to have had microscopically positive margins. Our findings mirror data reported by DeMatteo et al. ${ }^{7}$ who reported that patients undergoing NAR for CRLM had a higher R1 resection rate when compared with those who received AR. 
Fig. 2 Kaplan-Meier survival curves comparing recurrence-free survival in patients undergoing anatomic and non-anatomic hepatic resections for NELM

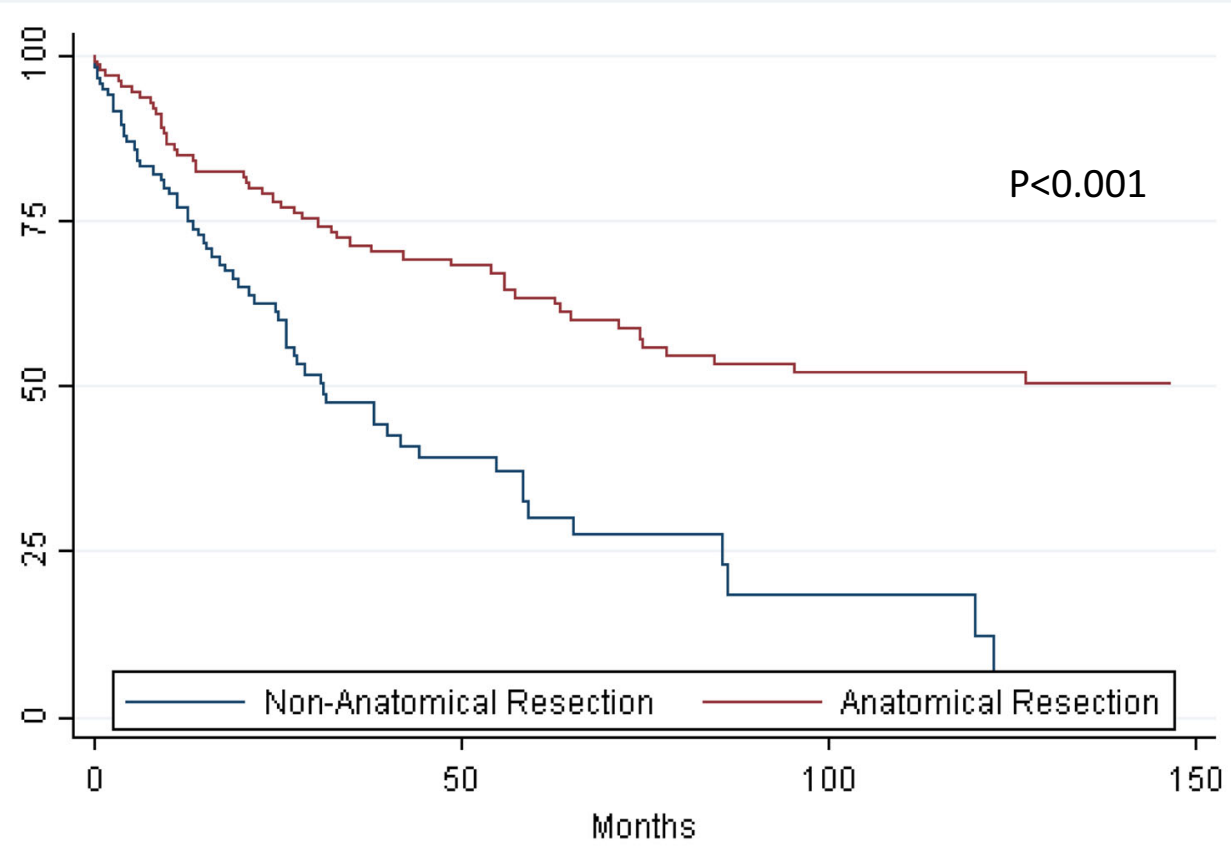

Furthermore, patients who had an $\mathrm{R} 1$ resection margin were independently more likely to experience death or recurrence as compared to patients who had a complete surgical R0 resection. This finding is in contrast to a 2010 study by Glazer et al. ${ }^{14}$ that reported on survival after surgical management of NELM. This difference may be explained in the extreme variability of treatment regimens offered and prescribed to patients with NELM. Furthermore, the study by Glazer et al. had a limited sample size and did not report on the type of surgical resection performed. Taken together, patients undergoing a NAR with curative intent are significantly more likely to have an incomplete surgical resection and subsequently are at higher odds of death and compared with patients who undergo an AR.

In the current study, the 1-, 3-, and 5-year OS and RFS were similar to the data reported in previous reports that evaluated long-term survival in patients with neuroendocrine tumors and hepatic metastases. ${ }^{15}$ However, to our knowledge, this is the first report evaluating the effect of AR vs. NAR when performed for NELM. Not surprisingly, higher pathologic tumor grade and lymph node metastases were associated with worsened survival. The presence of bilateral metastases and an estimated liver involvement of $\geq 50 \%$ were not associated with worse OS, a finding which is consistent with other series. ${ }^{16}$ This is likely due to the indolent nature of these tumors and the relative effectiveness of non-surgical treatment modalities (thermal ablation, transcatheter therapies, medical management, etc.) that can significantly augment a patient's disease course. On univariable analysis, type of liver resection was found to be associated with worsened OS; however, this significant difference was not maintained after multivariable hazard regression analysis. Conversely, after adjusting for covariates including tumor grade and resection margin status, patients who underwent an NAR saw a greater than 2-fold increased risk of recurrence when compared to those who underwent AR for their NELM. This finding could be related to several patient and tumor-related factors. First, in addition to margin status, patients undergoing NAR could be at increased risk for recurrence due to the potential micrometastatic disease removed in larger AR specimens. In addition, though the surgical margins may have been negative, there may have been a field defect in the nearby liver with other nearby areas of potential disease which may have not been clinically apparent at the time of surgery. Additionally, patient selection may have impacted the findings as surgeons may have disproportionally allocated NAR to patients with more aggressive disease biology. This was not reflected in the available data, however, as tumor grade, location, and percent liver involvement were all controlled for in the analysis. Collectively, it appears that patients who undergo NAR appear to be at higher risk of recurrence as compared to patients who undergo AR for NELM.

The current study has several limitations. As with all retrospective studies, uncontrolled treatment allocation bias is a distinct and likely possibility. As previously addressed, patients with aggressive tumor biology or other uncontrolled factors may have been represented disproportionately in the data. Furthermore, patients in the current cohort were also treated at high-volume international hepatobiliary centers, which may limit the generalizability of the results. Finally, certain clinicopathologic data (e.g., Ki-67, exact tumor location) and post-operative data (e.g., complications) were not collected and therefore these variables could not be assessed. 
In conclusion, patients with NELM experience reasonable long-term survival following curative intent surgical resection. Patients who undergo NAR are at an independent higher risk of NELM recurrence. Overall survival, however, does not appear to be impacted by extent of liver resection for NELM.

\section{Compliance with Ethical Standards}

The Institutional Review Board of the participating institutions deemed this study exempt for review.

\section{References}

1. Spolverato G, Bagante F, Wagner D, et al. Quality of life after treatment of neuroendocrine liver metastasis. J Surg Res. 2015;198(1):155-164.

2. Thompson GB, van Heerden JA, Grant CS, Carney JA, Ilstrup DM. Islet cell carcinomas of the pancreas: a twenty-year experience. Surgery. 1988;104(6):1011-1017.

3. Mayo SC, de Jong MC, Pulitano C, et al. Surgical management of hepatic neuroendocrine tumor metastasis: results from an international multi-institutional analysis. Ann Surg Oncol. 2010;17(12): 3129-3136.

4. Farley HA, Pommier RF. Treatment of Neuroendocrine Liver Metastases. Surg Oncol Clin N Am. 2016;25(1):217-225.

5. Tang H, Li B, Zhang H, Dong J, Lu W. Comparison of Anatomical and Nonanatomical Hepatectomy for Colorectal Liver Metastasis: A Meta-Analysis of 5207 Patients. Sci Rep. 2016;6:32304.

6. Margonis GA, Buettner S, Andreatos N, et al. Anatomical Resections Improve Disease-free Survival in Patients With
KRAS-mutated Colorectal Liver Metastases. Ann Surg. 2017;266(4):641-649.

7. DeMatteo RP, Palese C, Jarnagin WR, Sun RL, Blumgart LH, Fong Y. Anatomic segmental hepatic resection is superior to wedge resection as an oncologic operation for colorectal liver metastases. $J$ Gastrointest Surg. 2000;4(2):178-184.

8. Lee SY, Cheow PC, Teo JY, Ooi LL. Surgical treatment of neuroendocrine liver metastases. Int J Hepatol. 2012;2012:146590.

9. Musunuru S, Chen H, Rajpal S, et al. Metastatic neuroendocrine hepatic tumors: resection improves survival. Arch Surg. 2006;141(10):1000-1004; discussion 1005.

10. Ejaz A, Reames BN, Maithel S, et al. Cytoreductive debulking surgery among patients with neuroendocrine liver metastasis: a multi-institutional analysis. HPB (Oxford). 2017.

11. Ejaz A, Reames BN, Maithel S, et al. The impact of extrahepatic disease among patients undergoing liver-directed therapy for neuroendocrine liver metastasis. J Surg Oncol. 2017;116(7):841-847.

12. Klimstra DS, Modlin IR, Coppola D, Lloyd RV, Suster S. The pathologic classification of neuroendocrine tumors: a review of nomenclature, grading, and staging systems. Pancreas. 2010;39(6):707-712.

13. Touzios JG, Kiely JM, Pitt SC, et al. Neuroendocrine hepatic metastases: does aggressive management improve survival? Ann Surg. 2005;241(5):776-783; discussion 783-775.

14. Glazer ES, Tseng JF, Al-Refaie W, et al. Long-term survival after surgical management of neuroendocrine hepatic metastases. $H P B$ (Oxford). 2010;12(6):427-433.

15. Lesurtel M, Nagorney DM, Mazzaferro V, Jensen RT, Poston GJ. When should a liver resection be performed in patients with liver metastases from neuroendocrine tumours? A systematic review with practice recommendations. HPB (Oxford). 2015;17(1):17-22.

16. Hibi T, Sano T, Sakamoto Y, et al. Surgery for hepatic neuroendocrine tumors: a single institutional experience in Japan. Jpn J Clin Oncol. 2007;37(2):102-107. 